\section{P 42 STANDARDISED PARENTERAL NUTRITION PROVISION IN NEONATES MANAGED POSTOPERATIVELY FOR GASTROSCHISIS}

Paul-M. Windscheif, Rhoda Welsh, Kokila Lakhoo, Gillian Rivlin, Kenny McCormick. Oxford University Hospitals

10.1136/archdischild-2015-308634.50

Aim The aim of this study is to prospectively assess the effect of using standardised parenteral nutrition (TPN) in comparison to individualised TPN in neonates managed postoperatively for gastroschisis.

Method All neonates who had undergone surgical management for gastroschisis and received TPN were included in the study. Data was taken from a database system registered as a service evaluation tool with the local hospital.

Data on sixty one neonates (born with gastroschisis between Jan 2009 to Jun 2014) was recorded of which 30 had been on individualised TPN and 31 on standardised TPN. The standardised TPN was introduced as an equal alternative option to individualised TPN beginning of Dec 2011 with a starter formulation containing lower amount of electrolytes for first two days of life and a maintenance formulation with a fixed content per $100 \mathrm{ml}$ of glucose $12 \mathrm{~g}$ to $12.5 \mathrm{~g}$ and amino acid $3 \mathrm{~g}$, electrolytes, trace elements and vitamins. A standardised SMOF-lipid formulation with fat soluble vitamins was provided in a separate bag. The standardised TPN formulation had a stability of 21days, and was accessible on the ward 24 hours a day, 7 days a week.

All neonates (33 boys and 28 girls) had treatment with the above TPN (standardised 885 TPN days, median 15 days (range 7-66 days) of a total 1823 TPN days for a median of 30 days (range 7-199 days). The treatment started at median postnatal age of 24 hours (1 to 3 days). The median post-conceptual age was 35 weeks ( 28 to 37 weeks) and median birth weight was $2 \mathrm{~kg}$ ( 1.0 to $3.46 \mathrm{~kg}$ ). All neonates were monitored for continuous regular weight gain.

All the babies were also initially on antibiotics. 14 babies were on Intralipid (from which later 9 have been switched to Clinoleic, and 4 to SMOF), 2 on Clinoleic, and 45 on SMOF as lipid source.

Results from routine blood samples for electrolytes, alanine transferase (ALT), alkaline phosphatase (ALP), bilirubin, albumin, raised $\mathrm{C}$ reactive protein $(\mathrm{CRP})$ and blood stream infections were documented from the day of TPN initiation up to the establishing of oral feeds.

Results Five (17\%) neonates on standardised TPN needed to be managed temporarily with individualised TPN formulation (mean 4 days, range 1-14 days). 12 (19.6\%) temporarily had a growth of an organism (8 with coagulase negative staphylococcus) in the blood stream (5 on standardised TPN). 23 (37\%) neonates had temporarily raised CRP (equal number on standardised and individual TPN). 5 (8\%) had temporarily increased ALT (3 on standardised TPN). ALP increased during the TPN course for all neonates, $10(17 \%)$ beyond the factor of 4 (equal number on standardised and individual TPN, mean 7.6 range 4.4-11.8). One neonate had brief post-operative acute renal impairment but not related to TPN. Levels for sodium, potassium, phosphate, urea and albumin have not been significantly out of normal range for age for any neonate during the management with either individualised or standardised TPN. 8 (13\%) had increasing bilirubin $(>100 \mathrm{umol} / \mathrm{L})$ after first week (equal number on standardised and individual TPN).

Conclusion Standardised parenteral nutrition provision in neonates managed postoperatively for gastroschisis is a promising safe option and does not seem to adversely affect monitored parameters any more than individualised TPN. 